\title{
Prevalence and seroepidemiology of Haemophilus parasuis in Sichuan province, China
}

\author{
Zhenghao Wang $^{1}$, Qin Zhao $^{1}{ }^{1}$, Hailin Wei ${ }^{1}$, Xintian Wen ${ }^{1}$, Sanjie Cao ${ }^{1}$, Xiaobo Huang ${ }^{1}$, Rui Wu ${ }^{1}$, Qigui \\ Yan ${ }^{1}$, Yong Huang ${ }^{1}$, Yiping Wen Corresp. 1 \\ ${ }^{1}$ College of Veterinary Medicine, Sichuan Agricultural University, Chengdu, China \\ Corresponding Author: Yiping Wen \\ Email address: 100329erga@sina.com
}

Haemophilus parasuis, the causative agent of Glässer's disease, has been reported as widespread, but little is known about its epidemiology in Sichuan province of China. The goal of our research is to reveal the prevalence and distribution of $H$. parasuis in this area. Sampling and isolation were performed across Sichuan; isolates were processed using serotyping multiplex PCR (serotyping-mPCR) and agar gel diffusion (AGD) for confirmation of serovar identity. This study was carried out from January 2014 to May 2016 and $254 \mathrm{H}$. parasuis field strains were isolated from 576 clinical samples collected from pigs displaying clinical symptoms. The isolation frequency was $44.10 \%$. Statistically very significant differences of infection incidence were found in three age groups $(P<0.01)$ and different seasons $(P<0.01)$. Serovars $5(25.98 \%)$ and $4(23.62 \%)$ were the most prevalent, however, non-typeable isolates accounted for nearly $7.87 \%$. In terms of geographical distribution, serovars 5 and 4 were mostly prevalent in west and east Sichuan. The results confirmed that the combined approach was dependable and revealed the diversity and distribution of serovars in Sichuan province, which is vital for efforts aimed at developing vaccine candidates allowing for the prevention or control of $H$. parasuis outbreaks. 
1 Prevalence and seroepidemiology of Haemophilus parasuis in

\section{Sichuan province, China}

3 Zhenghao Wang ${ }^{1}$, Qin $\mathrm{Zhao}^{1}$, Hailin $\mathrm{Wei}^{1}$, Xintian Wen ${ }^{1}$, Sanjie $\mathrm{Cao}^{1}$, Xiaobo

4 Huang ${ }^{1}$, Rui $\mathrm{Wu}^{1}$, Qigui Yan ${ }^{1}$, Yong Huang ${ }^{1}$, Yiping Wen ${ }^{1}$

$5{ }^{1}$ College of Veterinary Medicine, Sichuan Agricultural University, Chengdu, Sichuan, China.

6 Corresponding Author

7 Yiping Wen

8 100329erga@sina.com 


\section{Abstract}

24

Haemophilus parasuis, the causative agent of Glässer's disease, has been reported as widespread, but little is known about its epidemiology in Sichuan province of China. The goal of our research is to reveal the prevalence and distribution of $H$. parasuis in this area. Sampling and isolation were performed across Sichuan; isolates were processed using serotyping multiplex PCR (serotyping-mPCR) and agar gel diffusion (AGD) for confirmation of serovar identity. This study was carried out from January 2014 to May 2016 and 254 H. parasuis field strains were isolated from 576 clinical samples collected from pigs displaying clinical symptoms. The isolation frequency was $44.10 \%$. Statistically very significant differences of infection incidence were found in three age groups $(P<0.01)$ and different seasons $(P<0.01)$. Serovars $5(25.98 \%)$ and $4(23.62 \%)$ were the most prevalent, however, non-typeable isolates accounted for nearly $7.87 \%$. In terms of geographical distribution, serovars 5 and 4 were mostly prevalent in west and east Sichuan. The results confirmed that the combined approach was dependable and revealed the diversity and distribution of serovars in Sichuan province, which is vital for efforts aimed at developing vaccine candidates allowing for the prevention or control of $H$. parasuis outbreaks. 


\section{Introduction}

Haemophilus parasuis, a member of the family Pasteurellaceae, not only colonises in the upper

respiratory tract of healthy pigs during different breeding periods, but also emerges as the aetiological agent of Glässer's disease in some cases (Oliveira \& Pijoan, 2004). This organism incurs significant economic losses within the swine industry. Therefore, efforts have been in studying the epidemiology and pathogenesis of this microbe. So far, 15 serovars and a considerable number of non-typeable isolates have been identified. Varous molecular approaches have revealed the diversity in genotypic lineage and have shed light on the heterogeneity of pathogenic H. parasuis serovars (Olvera et al., 2006; Del Río et al., 2006; Zhang et al., 2011). Traditionally, agar gel diffusion (AGD) and indirect hemagglutination (IHA) are used to serotype H. parasuis field isolates (Kielstein \& Rapp-Gabrielson, 1992; Del Río et al., 2003a). In addition, the latter was once proposed as a more discriminatory method for improving the rate of serotyping (Turni \& Blakall, 2005). Subsequently, a multiplex PCR was reported for rapid molecular serotyping based on the analysis of variation within the capsule loci (Howell et al., 2015). Each serovar has its own distinctive markers except serovars 5 and 12, for they share the same marker. AGD using specific hyperimmune antisera may be applied to remedy the serotyping issues seen in distinguishing between serovars 5 and 12 . 
62

63

64

production of cross-protecting vaccines among different serovars has met with little success (Rapp-Gabrielson et al., 1997; Brockmeier et al., 2013). Commercial vaccines for use in swine seldom match the causative serovars and inoculation tends to be ineffective; this illustrating the fact the commercial vaccines have, thus far, proven incapable of serovar cross protection in China. Detailed information of morbidity and seroepidemiology can play an important role in prevention when applied in conjuction with research aimed at uncovering potential antigenic markers that are highly conserved across multiple serovars. As no detailed report of $H$. parasuis infection was available in the Sichuan province, China, we investigated the prevalence and seroepidemiology of H. parasuis in Sichuan province from January 2014 to May 2016.

\section{Materials and methods}

\section{Reference strains and hyperimmune antisera}

Reference strains of serovars 5 and 12 were purchased from Bacteriology Research Laboratory, Animal Research Institute, Yeerongpilly 4105, Australia. Subsequently, corresponding hyperimmune reference antisera of rabbit source were prepared (Kielstein \& Rapp-Gabrielson, 1992).

\section{Collection of samples}

From January 2014 to May 2016, a total of 576 clinical samples were collected from 576 weak or moribund pigs showing respiratory distress or arthritis. The survey focused on 103 intensive swine farms in the Sichuan province. The farms covered 28 counties of 13 cities of Sichuan province. Incidentally, the province was divided into two parts artificially by climate rather than vertical division. The annual average temperature of the northeast region was a bit higher than 
83

84

85

86

87

88

89

90

91

92

that of the southwest region in Sichuan province.

All pigs were necropsied humanely. Lungs, heart blood, joints and brain were derived from pigs showing signs of acute infection or septicemia, while lungs, pericardium liquid, pleural effusion, seroperitoneum and joint fluid as well as fibrinous membrane were collected from pigs displaying relatively mild symptoms (Turni \& Blackall, 2007). At least two tissues or exudates per pig were analyzed in the present study. Different tissues or exudates from the same pig were considered to be one sample. Additionally, all samples were classified by age groups, seasons and regions.

\section{Isolation and identification}

Samples were inoculated on tryptic soy agar (TSA, Becton, Dickinson and Company) with a final concentration of $5 \%$ newborn calf serum and $1 \mu \mathrm{g} / \mathrm{ml}$ of nicotinamide adenine dinucleotide (NAD, Roche). The plates were incubated for at least $24 \mathrm{~h}$ at $37^{\circ} \mathrm{C}$ under aerobic conditions.

Colonies with translucent and 1mm-diameter size were further passaged. Subsequently, Gram staining was carried out and culture with a typical morphology of H. parasuis such as threadless and medium or short rod was confirmed by PCR as described elsewhere (Oliveira et al., 2001). Moreover, strains isolated from different tissues of a pig were counted as one isolate.

\section{Serotyping}

First, all the isolates were serotyped by multiplex PCR using molecular markers and reaction procedure as previously described (Howell et al., 2015). As noted previously, AGD, was performed to differentiate between serovars 5 and 12. Pure cultures of the isolates with the marker of serovars 5 and 12 were grown in tryptic soy broth (TSB, Becton, Dickinson and 
104 Company) and cells were harvested by centrifugation at under $8,000 \times \mathrm{g}$ for $5 \mathrm{~min}$, and then

105 resuspended in phosphate buffered saline (pH: 7.4); cell concentration was adjusted

$106\left(\mathrm{OD}_{600}=1.2 \pm 0.1\right)$. The cells was heated at $121^{\circ} \mathrm{C}$ for $2 \mathrm{~h}$ and followed by a centrifugation under

$1076,000 \times \mathrm{g}$ for $5 \mathrm{~min}$ and supernatant was collected as heat-stable antigen. Using the hyperimmune

108 antisera against serovars 5 and 12 earlier prepared, AGD was performed with 3\% agarose by

109 using heat-stable antigens of standard strains as positive controls, ultrapure water as a blank

110 control and Actinobacillus pleuropneumoniae as a negative control. A single immune line

111 between antiserum holes and heat-stable antigen holes indicated positives. Strains of the same

112 serovar from a single farm were counted as one isolate.

113 Statistical analysis

114 Data was statistically analyzed by the procedure of IBM SPSS Statistics 19.0, and differences

115 between age groups, seasons and regions were performed by Chi-square analysis. All tests were

116 2-sided and $P<0.01$ was defined as a very significant difference while $P<0.05$ was defined as a

117 significant difference.

118 Results

119 Bacterial strains

120 In the present investigation, varying degrees of respiratory symptom and classic Glässer's

121 disease were found in herds of the intensive swine farms (Fig. 1). 254 field H.parasuis isolates

122 were confirmed via viewing culture morphology characteristics and 16S rRNA PCR results, with

123 the isolation rate of $44.10 \%$. The infection incidence of $H$. parasuis was $45.98 \%$ and $43.77 \%$ in

1242014 and 2015 , respectively, and $41.10 \%$ was measured in the first five months of 2016 , 
125 showing no significant differences $\left(\chi^{2}=0.531, P>0.05\right)$. However, infection incidence varied 126 with age, season and region. In pigs of 3-8 weeks, the general infection incidence was $60.80 \%$, 127 which was much higher than that of pigs of less than 3 weeks or over 8 weeks. Statistically very 128 significant differences were found in three age groups as whole $\left(\chi^{2}=108.209, P<0.01\right)$.

Moreover, infection incidence in winter and spring were much higher than the other two seasons, and statistical differences were very significant $\left(\chi^{2}=27.854, P<0.01\right)$ which indicated winter and spring were the major epidemic seasons of H.parasuis in Sichuan province. However, no significant differences were seen in two regions as whole $\left(\chi^{2}=3.031, P>0.05\right)$ (Table 1$)$.

\section{mPCR and AGD for serotyping}

134 Follow-up serotype-mPCR and complementary AGD examination classified them into 11 serovars, which showed a considerable serovar diversity (Fig. 2; Fig. 3). Serovar 5 (66 of 254, $25.98 \%)$ and serovar 4 (60 of 254, 23.62\%) were most prevalent. However, serovars 3, 6, 10 and

13715 were not found. The typeable and non-typeable isolates were $234(92.13 \%)$ and $20(7.87 \%)$ out of total 254 respectively.

\section{Distribution and tendency of serovars in Sichuan province}

In the present study, geographical distribution of serovars indicated that serovars 5 and 4 were the most prevalent in Sichuan province (Fig. 4A). In addition, serovars 1, 7 and 12 were only found in west Sichuan and serovar 8 was only found in east Sichuan (Fig. 4A). On the other hand, serovars 4 and 5 were prevalent in both 2014 and 2015, however, serovar 7 was the lead in prevalence for the first five months of 2016 (Fig. 4B).

\section{DISCUSSION}


146 Isolation and identification of $H$. parasuis is the "gold standard" for diagnosis, but it requires that

147 the samples to be collected from acute or typical infection cases without antimicrobial treatment

148 as possible. Furthermore, rigorous nutritional demands and fragility of $H$. parasuis slows down

149 the culture process and increases the probability of false negatives (Del Río et al., 2003b). The

150 isolation rate of previous studies ranged from $0.99 \%$ to $21 \%$ (Oliveira et al., 2003; Fablet et al.,

151 2012; Zhang et al., 2012). In the present study, the isolation rate was $44.10 \%$ in the Sichuan

152 province (Table 1), and revealed a mark differences in prevalence in different areas. Differences

153 of infection incidence among different age groups were in accordance with previous reports,

154 which reconfirmed that $H$. parasuis infection was a big threat to piglets. In addition, an

155

156

157

158

159

160

161

162

163

164

165

166

apparently increased infection rate in winter and spring was due mainly to the sudden change of temperature and poor ventilation.

Before 2015, studies on epidemiology of $H$. parasuis usually depended on traditional approaches for serotyping. In 2012, 112 (out of 536 pigs) $H$. parasuis strains were isolated in southern China, and combination of AGD and IHA revealed that serovars 5 and 4 were the most prevalent serovars, whereas non-typeable strains accounted for nearly 20\% (Zhang et al., 2012). In Northern Italy, serovars 4, 13 and 5 were demonstrated as the top three serovars in epidemic $H$. parasuis strains using AGD, however, non-typeable strains accounted for 27.3\% (Luppi et al., 2013). The simplex method is always subjected to susceptibility and improper preparations of hyperimmune antisera. By combination of $\mathrm{MPCR}$ and complementary AGD, the profile of prevalent serovars in the Sichuan province has been revealed in the present study (Fig. 4), which confirmed that the combined approach proposed here is efficient and reliable. Considering the 
167 existence of non-typeable strains, the most convincing hypothesis is that there is deletion or

168 insertion or mutation in serotype specific genes on the capsule loci. Secondly, there are

169 differences between phenotypic and genotypic serotype in certain bacteria due to other

170 alterations (Gentle et al., 2016). According to the previous report, the two in silico-nontypeable

171 isolates can be differentiate by mPCR which indicated a non-absolute concordance between

$172 \mathrm{mPCR}$ and in silico serotype analysis. We also found that some strains cannot be identified by

173 mPCR, but the in silico serotype analysis is able to differrntiate them (Howell et al., 2015). Thus,

174 more efforts have to be made to elucidate the differences. Moreover, some serovars previously

175 believed to be nonvirulent or hypervirulent were also isolated with a frequency of $18.89 \%$, and

176 the discrepancy is due, to some extent, to the immune capacities, feeding conditions and

177 supervision, and so forth (Kielstein \& Rapp-Gabrielson, 1992; Brockmeier et al., 2013; Yu et al., 178 2014).

There are four bivalent and one monovalent inactivated vaccines for $H$. parasuis authorized in mainland China, however, desired protection are seldom obtained on account of the poor cross protection among these endemic serovars. Therefore, vaccines corresponding to specific serovars fits the region are optimal (Yu et al., 2014). However, no recent seroepidemiology reports were accessible for the Sichuan province and the prevalent serovars may change over time. The serovar profile in the present investigation was partly consistent with previous reports in China mainland, however, following serovars 4 and 5 were serovars 7, 1 and 2 rather than serovars 14, 13 and 12 as previously reported (Cai et al., 2005; Zhang et al., 2012; Chen et al., 2015).

Furthermore, despite the most two epidemic serovars being the same in the two parts of the 
188 Sichuan province, the secondary serovars may interfere in the control of $H$. parasuis infection.

189 The present study revealed the seroepidemiology of $H$. parasuis in Sichuan province, China.

190 Thus, existing vaccines and vaccine candidate strains should be taken into consideration to

191 control the disease. To our knowledge, several vaccine manufacturers and laboratories were

192 considering more appropriate adjuvant candidates to reduce the irritation generated by

193 inactivated vaccines. Furthermore, genetically engineered and attenuated vaccines against

194 virulent strains of different serovars might be promising (Oliveira et al., 2003). Although the

195 prevalent serovars in the present study in a given area may change over a prolonged time span,

196 throughout a two and a half year period, we believe that the serovars percentage profiles

197 remained stable. Subsequent investigation should be carried out on annual basis to understand

198 the variation tendency in seroepidemiology of H.parasuis in Sichuan province.

199 CONCLUSION

200 This study confirmed that there was a high infection rate of H.parasuis in Sichuan province from

201 January 2014 to May 2016. The present research revealed that H. parasuis incidence was the

202 highest in nursery pigs and infection rates in winter and spring were much higher than during the

203 other two seasons. In addition, it is also demonstrated serovars 4 and 5 were the most prevalent

204 in the Sichuan province, and mPCR combined with AGD was dependable for H. parasuis

205 serotyping.

206 ACKNOWLEDGEMENTS

207 We thank the collaborating producers for facilitating the study on their farms, and thank the members of the Swine Disease Center of the College of Veterinary Medicine for their underlying 
209

210

211

212

213

contributions. We especially thank Prof. Yung-Fu Chang of Cornell University for the critical reading and edition of this manuscript.

\section{REFERENCES}

Brockmeier SL, Loving CL, Mullins MA, Register KB, Nicholson TL, Wiseman BS, Baker RB, Kehrli ME. 2013. Virulence, transmission, and heterologous protection of four isolates of Haemophilus parasuis. Clinical and Vaccine Immunology 20(9):1466-1472. DOI 10.1128/CVI.00168-13.

Cai X, Chen H, Blackall PJ, Yin Z, Wang L, Liu Z, Jin M. 2005. Serologicalcharacterization of Haemophilus parasuis isolates from China. Veterinary Microbiology 111(3-4):231-236 DOI 10.1016/j.vetmic.2005.07.007.

Chen S, Chu Y, Zhao P, He Y, Jian Y, Liu Y, Lu Z. 2015. Development of arecombinant OppA-based indirect hemagglutination test for the detection ofantibodies against Haemophilus parasuis. Acta Tropica 148:8-12 DOI 10.1016/j.actatropica.2015.04.009.

Del Río ML, Martín CB, Navas J, Gutiérrez-Muñiz B, Rodríguez-Barbosa JI, Rodríguez Ferri EF. 2006. aroA gene PCR-RFLP diversity patterns in Haemophilus parasuis and Actinobacillus species. Research in Veterinary Science 80(1):55-61 DOI 10.1016/j.rvsc.2005.03.004.

Del Río ML, Gutiérrez CB, Rodríguez Ferri EF. 2003. Value of Indirect Hemagglutination and Coagglutination Tests for Serotyping Haemophilus parasuis. Journal of Clinical Microbiology 41(2): 880882.

Del Río ML, Gutiérrez B, Gutiérrez CB, Monter JL, Rodríguez Ferri EF. 2003. Evaluation of survival of Actinobacillus pleuropneumoniae and Haemophilus parasuis in four liquid media and two swab specimen transport systems. American Journal Veterinary Research 64(9):1176-1180 
230

231

232

233

234

235

236

237

238

239

240

241

242

243

244

245

246

247

248

249

250

Fablet C, Marois C, Dorenlor V, Eono E, Eveno E, Jolly JP, Le Devendec L, Kobisch M, Madec F, Rose

N. 2012. Bacterial pathogens associated with lung lesions in slaughter pigs from 125 herds. Research in

Veterinary Science 93(2):627-630 DOI 10.1016/j.rvsc.2011.11.002.

Gentle A, Ashton PM, Dallman TJ, Jenkins C. 2016. Evaluation of molecular methods for serotyping Shigella flexneri. Journal of Clinical Microbiology 54(6):1456-1461 DOI 10.1128/JCM.03386-15.

Howell KJ, Peters SE, Wang J, Hernandez-Garcia J, Weinert LA, Luan SL, Chaudhuri RR, Angen Ø, Aragon V, Williamson SM, Parkhill J, Langford PR, Rycroft AN, Wren BW, Maskell DJ, Tucker

AW, BRaDP1T Consortium. 2015. Development of a multiplex PCR for rapid molecular serotyping of Haemophilus parasuis. Journal of Clinical Microbiology 53(12):3812-3821 DOI 10.1128/JCM.01991-15.

Kielstein P, Rapp-Gabrielson VJ. 1992. Designation of 15 serovars of Haemophilus parasuis on the basis of immunodiffusion using heat-stable antigen extracts. Journal of Clinical Microbiology 30(4):862-865

Luppi A, Bonilauri P, Dottori M, Iodice G, Gherpelli Y, Merialdi G, Maioli G, Martelli P. 2013. Haemophilus parasuis serovars isolated from pathological samples in Northern Italy. Transboundary and Emerging Disease 60(2):140-142 DOI 10.1111/j.1865-1682.2012.01326.x.

Oliveira S, Pijoan C. 2004. Haemophilus parasuis : new trends on diagnosis, epidemiology and control. Veterinary Microbiology 99(1):1-12 DOI 10.1016/j.vetmic.2003.12.001

Olvera A, Calsamiglia M, Aragon V. 2006. Genotypic diversity of Haemophilus parasuis field strains. Applied and Environmental Microbiology 72(1):3984-3992 DOI 10.1128/AEM.02834-05.

Oliveira S, Galina L, Pijoan C. 2001. Development of a PCR test to diagnose Haemophilus parasuis infections. Journal of Veterinary Diagnostic Investigation 13(6):495-501

Oliveira S, Blackall PJ, Pijoan C. 2003. Characterization of the diversity of Haemophilus parasuis field 
251

252

253

254

255

256

257

258

259

260

261

262

263

264

265

266

267

268

269

270

271

isolates by use of serotyping and genotyping. American Journal of Veterinary Research 64(4):435-442.

Rapp-Gabrielson VJ, Gordon JK, Jeffrey TC, Stephen KM. 1997. Haemophilus parasuis: Immunity in swine after vaccination. Veterinay Medicine 92:83-90.

Turni C, Blackall PJ. 2005. Comparison of the indirect haemagglutination and gel diffusion test for serotyping Haemophilus parasuis. Veterinary Microbiology 106(1-2):145-151 DOI

10.1016/j.vetmic.2004.12.019

Turni C, Blackall PJ. 2007. Comparison of sampling sites and detection methods for Haemophilus parasuis. Australian Veterinary Journal 85(5):177-184 DOI 10.1111/j.1751-0813.2007.00136.x

Yu J, Wu J, Zhang Y, Du Y, Peng J, Chen L, Sun W, Cong X, Xu S, Shi J, Li J, Huang B, Zhu X, Wang

J. 2014. Identification of putative virulence-associated genes among Haemophilus parasuis strains and the virulence difference of different serovars. Microbial Pathogenesis 77:17-23 DOI

10.1016/j.micpath.2014.10.001

Zhang J, Xu C, Guo L, Shen H, Deng X, Ke C, Ke B, Zhang B, Li A, Ren T, Liao M. 2012. Prevalence and characterization of genotypic diversity of Haemophilus parasuis isolates from southern China. Canadian Journal of Veterinary Research 76(3):224-229

Zhang J, Xu C, Guo L, Ke B, Ke C, Zhang B, Deng XL, Liao M. 2011. A rapid pulsed-field gel electrophoresis method of genotyping Haemophilus parasuis isolates. Letters in Applied Microbiology 52(6):589-595 DOI 10.1111/j.1472-765X.2011.03048.x 


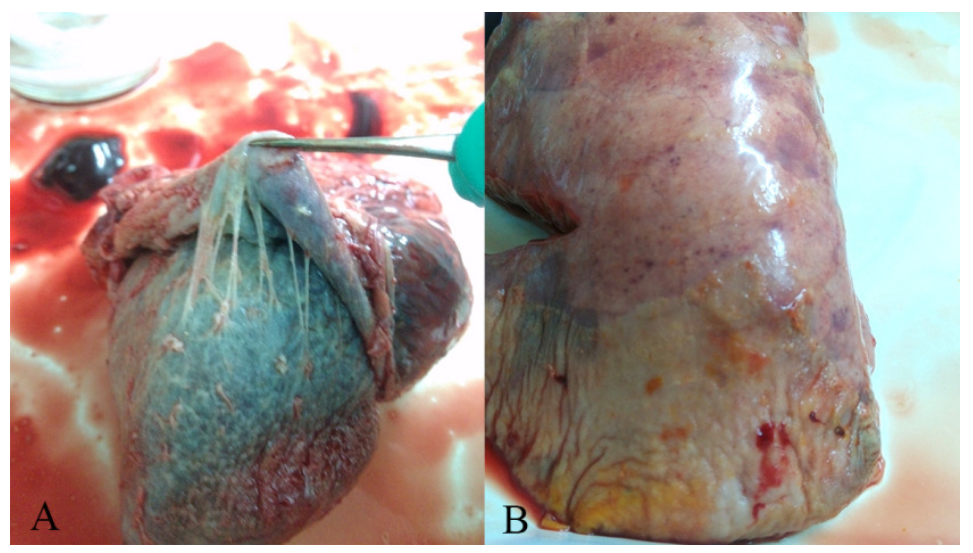

Figure 1 Gross lesions in $\boldsymbol{H}$. parasuis infected pigs. (A) Fibrinous pericarditis. (B) Fibrinous pleuritis.

282

283

284

285

286

287 
Table 1 Haemophilus parasuis infections in Sichuan province, China (January, 2014-May, 2016).

\begin{tabular}{|c|c|c|c|c|c|c|c|c|c|c|}
\hline \multirow{2}{*}{$\begin{array}{l}\text { Yea } \\
\text { r }\end{array}$} & \multirow{2}{*}{ Total } & \multicolumn{3}{|c|}{ Age groups (weeks) } & \multicolumn{4}{|c|}{ Seasons } & \multicolumn{2}{|c|}{ Regions } \\
\hline & & $<3$ & $3-8$ & $>8$ & winter & spring & summer & autumn & east & west \\
\hline 2014 & $80 / 174^{*}$ & $2 / 24$ & $65 / 104$ & $13 / 46$ & $29 / 61$ & $45 / 86$ & $6 / 19$ & $0 / 8$ & $20 / 45$ & $60 / 129$ \\
\hline 2015 & $144 / 329$ & $3 / 51$ & $120 / 186$ & $21 / 92$ & $67 / 136$ & $52 / 93$ & $7 / 47$ & $18 / 53$ & $32 / 83$ & $112 / 246$ \\
\hline 2016 & $30 / 73$ & $1 / 7$ & $29 / 62$ & $0 / 4$ & $9 / 25$ & $21 / 48$ & / & / & $0 / 10$ & $30 / 63$ \\
\hline Tota & $254 / 576$ & $6 / 82$ & $214 / 352$ & $34 / 142$ & $105 / 222$ & $118 / 22$ & $13 / 66$ & $18 / 61$ & $52 / 138$ & $202 / 438$ \\
\hline 1 & & & & & & 7 & & & & \\
\hline
\end{tabular}

*: A/B, A represents number of strains, and B represents number of samples.

300

301

302 


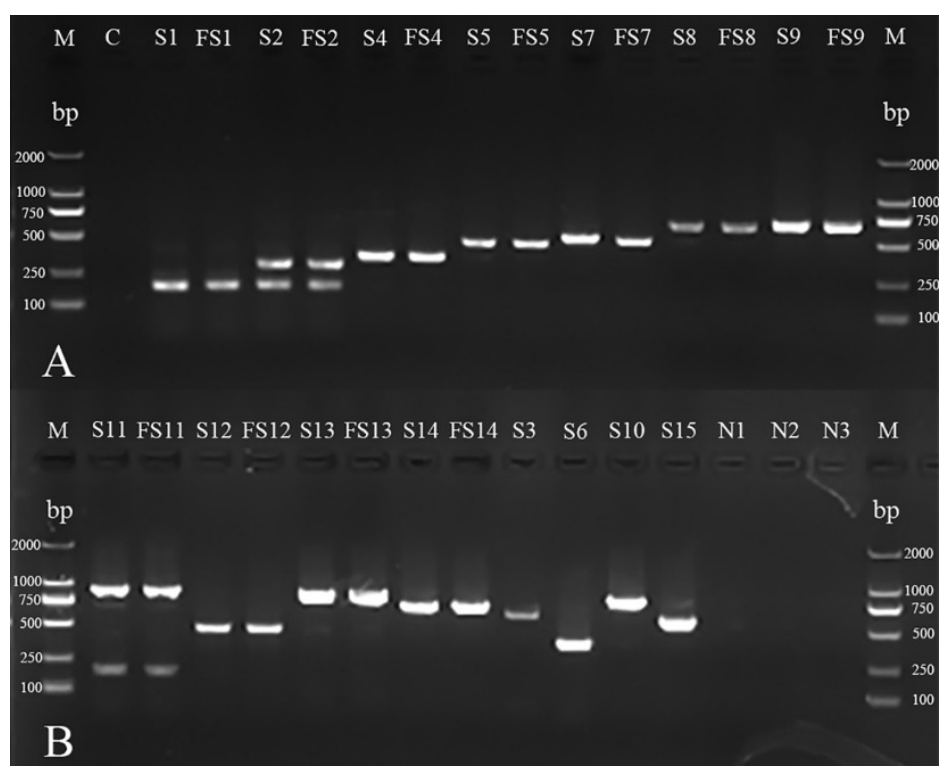

Figure 2 mPCR of serotyping for $\boldsymbol{H}$. parasuis field isolates and standard strains. (A) M: DL 2000 DNA

Marker (Takara); C: blank control; S1, S2, S4, S5, S7, S8 and S9: standard strains of serovars 1, 2, 4, 5, 7, 8 and 98; FS1, FS2, FS4, FS5, FS7, FS8 and FS9: field isolates AB14011, GA14051, YA14041, GA14032, 
318 LS14031, GA15013, NC14041 (There were no field isolates of serovars 3, 6, 10 or 15 obtained in the

319 investigation). (B) M: DL 2000 DNA Marker (Takara); S11, S12, S13, S14, S3, S6, S10 and S15: standard

320 strains of serovars 11, 12, 13, 14, 3,6,10 and 15; FS11, FS12, FS13 and FS14: field isolates CD14021,

321 LS15044, NJ14021, MY14021; N1: non-typeable strain; N2: A. pleuropneumoniae; N3: Streptococcus suis.

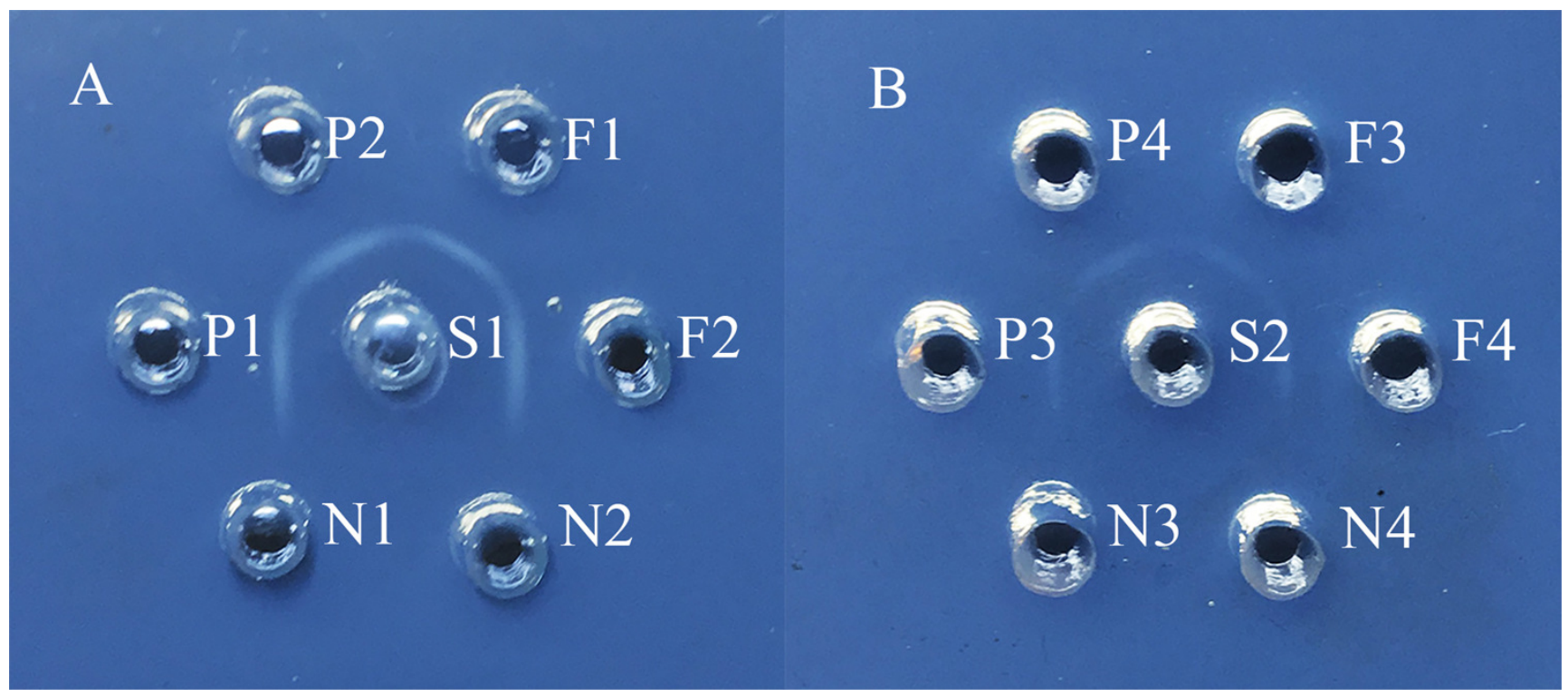

Figure 3 Agar gel diffusion (AGD) of serotyping for $\boldsymbol{H}$. parasuis field isolates. (A). S1: hyperimmune

antiserum of serovar 5; F1: H. parasuis field isolates GA14032; F2: H. parasuis field isolates CD14041; P1 and P2: positive control of reference strain for serovar 5; N1: negative control of A. pleuropneumoniae; N2: 
333

334

335

336

337

338

339

340

341

342

343

344

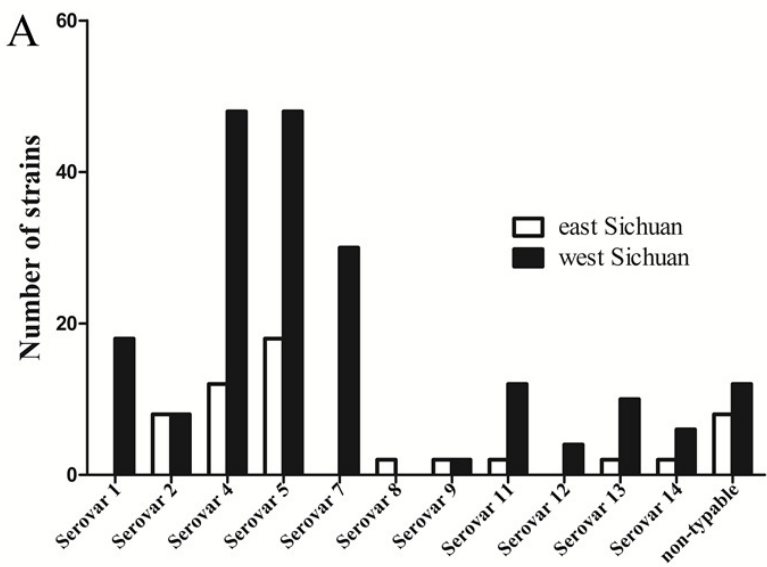

345

346

347 control of A. pleuropneumoniae; N4: blank control.

6

7

8

39

42

43

Serovars

of $H$. parasuis during a two year, five months survey period.

blank control. (B). S2: hyperimmune antiserum of serovar 12; F3: H. parasuis field isolates CD14051; F4: $H$.

parasuis field isolates LS15044; P3 and P4: positive control of reference strain for serovar 12; N3: negative

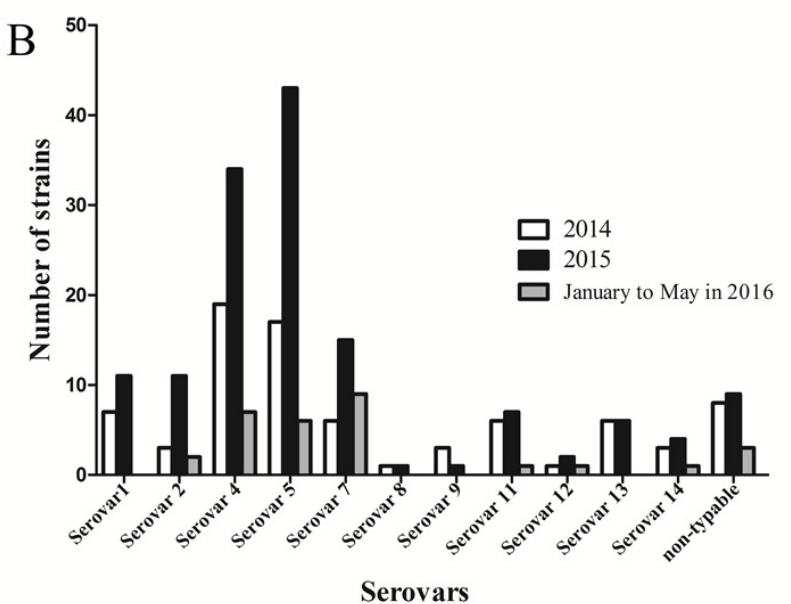

Serovars

Figure 4: Distribution and variation of different serovars of $\boldsymbol{H}$.parasuis in Sichuan province, China. (A)

Distribution of different serovars of $H$. parasuis in east and west Sichuan. (B) Variation of different serovars 